\title{
Probing the Energy Conversion Pathways between Light, Carriers, and Lattice in Real Time with Attosecond Core-Level Spectroscopy
}

\author{
T. P. H. Sidiropoulos $\odot,{ }^{1, \dagger}$ N. Di Palo, ${ }^{1, \dagger}$ D. E. Rivas $\odot,{ }^{1,2, \dagger}$ S. Severino, ${ }^{1}$ M. Reduzzi, ${ }^{1}$ B. Nandy, ${ }^{1}$ B. Bauerhenne $\odot,{ }^{3}$ \\ S. Krylow $\odot,{ }^{3}$ T. Vasileiadis $\odot,{ }^{4}$ T. Danz $\odot,{ }^{5}$ P. Elliott,${ }^{6,7}$ S. Sharma, ${ }^{6}$ K. Dewhurst ${ }^{7}$ C. Ropers, ${ }^{5}$ \\ Y. Joly, ${ }^{8}$ M. E. Garcia, ${ }^{3}$ M. Wolf, ${ }^{4}$ R. Ernstorfer®, ${ }^{4}$ and J. Biegert ${ }^{1,9}, *$ \\ ${ }^{1}$ ICFO-Institut de Ciencies Fotoniques, The Barcelona Institute of Science and Technology, \\ 08860 Castelldefels (Barcelona), Spain \\ ${ }^{2}$ European XFEL GmbH, Holzkoppel 4, 22869 Schenefeld, Germany \\ ${ }^{3}$ Theoretische Physik, FB-10, Universität Kassel, 34132 Kassel, Germany \\ ${ }^{4}$ Fritz Haber Institute of the Max Planck Society, 14195 Berlin, Germany \\ ${ }^{5} 4$ th Physical Institute-Solids and Nanostructures, University of Göttingen, 37077 Göttingen, Germany \\ ${ }^{6}$ Max-Born-Institut für Nichtlineare Optik und Kurzzeitspektroskopie, 12489 Berlin, Germany \\ ${ }^{7}$ Max-Planck-Institut für Mikrostrukturphysik, Weinberg 2, 06120 Halle, Germany \\ ${ }^{8}$ Université Grenoble Alpes, CNRS, Grenoble INP, Institut Néel, 38000 Grenoble, France \\ ${ }^{9}$ ICREA-Institució Catalana de Recerca i Estudis Avançats, 08010 Barcelona, Spain
}

(Received 9 June 2021; revised 2 September 2021; accepted 12 October 2021; published 27 December 2021; corrected 8 February 2022)

Detection of the energy conversion pathways between photons, charge carriers, and the lattice is of fundamental importance to understand fundamental physics and to advance materials and devices. Yet, such insight remains incomplete due to experimental challenges in disentangling the various signatures on overlapping timescales. Here, we show that attosecond core-level $\mathrm{x}$-ray absorption fine-structure spectroscopy (XANES) meets this challenge by providing an unambiguous and simultaneous view on the temporal evolution of the photon-carrier-phonon system. We provide surprising new results by applying the method to graphite, a seemingly well-studied system whose investigation is complicated by a variety of mechanisms occurring across a wide range of temporal scales. The simultaneous real-time measurement of electrons and holes reveals disparate scattering mechanisms for infrared excitation close to the Fermi energy. We find that ultrafast dephasing of the coherent carrier dynamics is governed by impact excitation (IE) for electrons, while holes exhibit a switchover from impact excitation to Auger heating (AH) already during the 11-fs duration of the infrared light field. We attribute this switchover to the limited scattering phase space in the $n$-doped material. We further elucidate the excitation mechanisms of strongly coupled optical phonons (SCOPs). The coherent excitation of both SCOPs is nondisplacive and is explained by the strong electron-phonon scattering, i.e., via a seemingly incoherent process. We identify the $A_{1}^{\prime}$ phonon as the dominating channel for dissipation of electronic coherence. Moreover, unobserved in graphite, we find high-frequency oscillations up to $90 \mathrm{THz}$, which arise from the modulation of the electronic density of states by the atomic displacements along the $E_{2 g}$ and $A_{1}^{\prime}$ modes. These measurements establish the utility of core-level XANES with attosecond temporal resolution to achieve an unambiguous and simultaneous view on the temporal evolution of the photon-carrier-phonon system with surprising new results even for a seemingly well-studied system like graphite. While the graphite measurement was conducted around the $K$ edge of carbon, adapting the methodology to other materials only requires spectra coverage of the respective elemental edge of the material's constituent. This flexibility makes our methodology widely applicable to detect and distinguish the various dynamic contributions to the flow of energy inside materials on their native timescales.

DOI: 10.1103/PhysRevX.11.041060

\author{
Subject Areas: Complex Systems \\ Condensed Matter Physics \\ Materials Science
}

\footnotetext{
*Corresponding author. jens.biegert@icfo.eu

${ }^{\dagger}$ These authors contributed equally to this work.

Published by the American Physical Society under the terms of the Creative Commons Attribution 4.0 International license. Further distribution of this work must maintain attribution to the author(s) and the published article's title, journal citation, and DOI. Open access publication funded by the Max Planck Society.
} 


\section{INTRODUCTION}

Investigation of the emergence of a specific response of a molecular or solid-state system requires the ability to resolve the quantum multibody dynamics between light, carriers, and nuclei [1]. Such investigation is still challenging due to the coupling between these various subsystems which occurs across vast temporal scales ranging from attoseconds into picoseconds; see Fig. 1(a). The subsystems are coupled since the electronic structure of a system arises from the electronic configuration of the individual nuclei of the system and their electrostatic and exchange interactions. Thus, a change in electronic occupation consequently leads to a change of nuclear position and vice versa. Independent of whether one investigates a solid-state or molecular system, most of the outstanding questions on the origin of a phenomenon, or physics effect, relate to nonadiabatic dynamics and to couplings between the different subsystems. The coupling itself further compounds the investigation, since the correlated interaction is not rigorously separable by individual measurements on different temporal and energetic scales. Without discrimination of the various mechanisms, however, it is difficult to understand when and why an excitation manifests adversely, for instance, as limited transport of charge carriers in metals [2,3], or as a reduced exciton lifetime in organic solids [4,5]. Conversely, it is important to understand why an excitation causes mutual attraction of fermion pairs [6], bosonic qubits [7], or how Floquet states can potentially stabilize high-temperature superconductivity [8] and induce topological quantum phases [9]. To address such canonical problems, methods are sought which provide a holistic view over how a light field creates a nonequilibrium state, how the state evolves into the

(a)

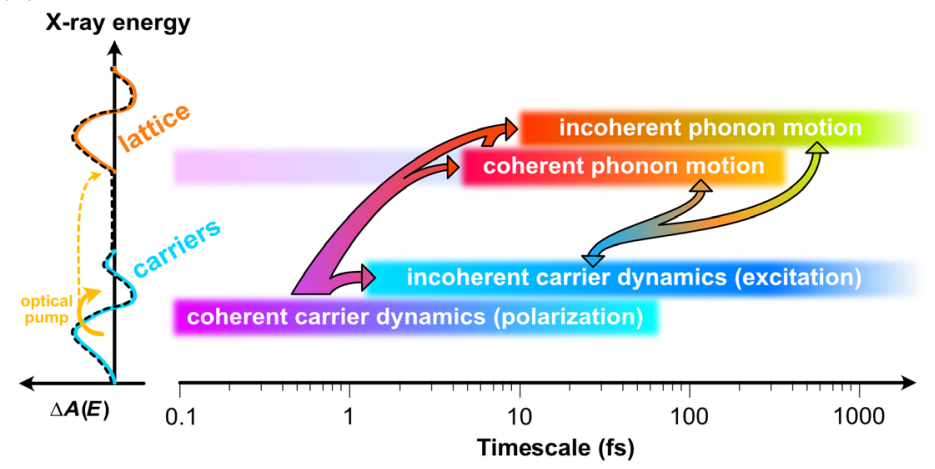

multibody state of excited electrons and holes, whether exciton pairs are stable, and which excitation of phonons leads to the relaxation of carriers and the lattice.

Among prominent methods to address this challenge, photoemission spectroscopies provide a detailed momentum-resolved insight into the electronic structure, but with limited depth of probing into the solid. Typically, the electronic coupling to the lattice can only be indirectly inferred [12], and with limited time resolution due to timebandwidth constraints $[13,14]$. In contrast, lattice dynamics are readily investigated with inelastic scattering methods such as Raman $[15,16]$, neutron, electron or x-ray scattering [17], and electron energy loss spectroscopy [18], but those methods only yield indirect information on the electronic structure. Evidently, each of these powerful methods has its own merits. But, to unravel the various, often intricated, contributions $[19,20]$ of carriers and phonons in solids [21,22], it is challenging, and many times even impossible [23,24], to combine the information from different methods to gain an understanding of the physics. Moreover, it is challenging to conduct measurements with different methods under identical conditions and on the same sample. It is thus highly desirable to extract carrier and lattice information from a single measurement.

\section{METHOD}

Here, we show that core-level $x$-ray absorption near edge structure (XANES) spectroscopy with attosecond soft $\mathrm{X}$-ray $(\mathrm{SXR})$ pulses $[25,26]$ meets the challenge in a single experimental method. The XANES method is based on the absorption of an x-ray photon whenever a dipole-allowed transition of a core-level electron is possible, either to a bound or to a continuum state. Core-level XANES is

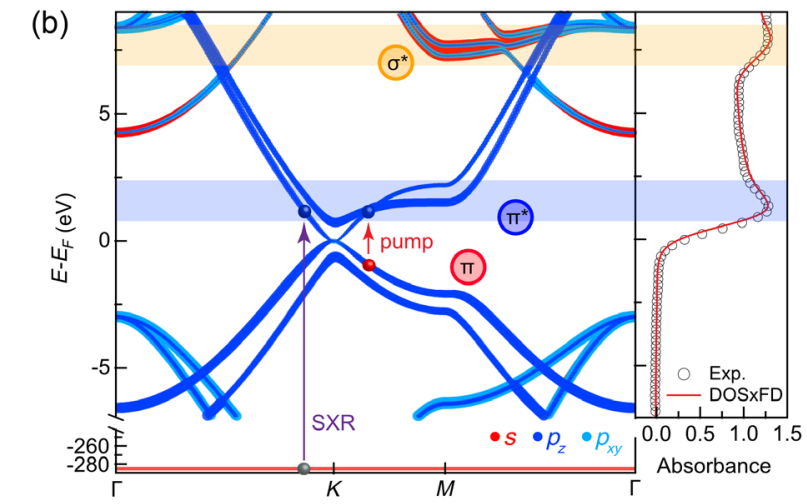

FIG. 1. Carrier and phonon dynamics in graphite. (a) The left-hand panel indicates two areas which are separated in x-ray photon energy, where changes in the differential absorption occur due to carrier and lattice motion. The right-hand panel indicates the vast time-overlapping physical effects and their interplay. (b) State-resolved band structure calculated in the local density approximation (LDA) with an ultrasoft Vanderbilt potential and Perdew-Burke-Ernzerhof (PBE) exchange correlation with QUANTUM ESPRESSO [10]. The blue and orange shaded areas indicate $\pi^{*}$ and $\sigma^{*}$ bands originating from $p_{z}$ and $s p_{2}$-hybridized states. The arrows show electronic transitions through SXR (violet) and near-infrared (red) pulses. The measured XANES spectrum (black circles), under $40^{\circ}$ incidence angle, is shown on the right. Electronic transitions from the carbon $1 s$ state (bound by $284.2 \mathrm{eV}$ ) to unoccupied states in both the $\pi^{*}$ and the $\sigma^{*}$ bands (1.5 and $7 \mathrm{eV}$ above the Fermi level) dominate the spectrum. The XANES spectrum is in excellent agreement with a numerical model (red) based on the density of states (DOS) and a Fermi-Dirac distribution at room temperature (see SM for further details [11]). 
element selective and orbital specific, and thus it is a sensitive probe of electronic structure. It is important to realize that unambiguous mapping of the XANES absorbance to the material's density of states (DOS) is strictly ensured only with $K$-shell XANES since the $1 s$ core transition does not suffer from final state multiplet effects [27]. This is distinct from measurements with extreme ultraviolet (attosecond) pulses, which access transitions from higher-lying core or innervalence states, and whose interpretation depends on the exact case and may require advanced theory. Figure 1(b) illustrates this point by showing that the absorbance from $K$-shell XANES unambiguously matches with the DOS calculated with density functional theory. Our conceptual approach is thus to time resolve the change in electronic structure of a material with attosecond $K$-shell XANES. To realize such measurement, it is vital to combine high-energy resolution with coverage of an extensive energy range of tens of $\mathrm{eV}$ to disentangle the various contributions to electronic structure change. Figure 1(a) shows this concept and indicates how the simultaneous measurement of energetically distinct signatures in the time-resolved XANES spectrum allows distinguishing electron, hole, and lattice dynamics. For instance, the attosecond time resolution allows detecting the buildup of coherences (polarization) even during the excitation light field's oscillation at terahertz to Petahertz $\left(10^{15} \mathrm{~Hz}\right)$ oscillation frequency. The spectral range within a few $\mathrm{eV}$ of the Fermi level records changes in electronic occupation. At the same time, alteration of the electronic structure (also) far from the Fermi level records nuclear motion.
We apply our methodology to graphite since the material is extensively studied and serves as a challenging benchmark due to the overlapping timescales of ultrafast carriercarrier $(c-c)$ and carrier-phonon $(c-\mathrm{ph})$ scattering, i.e., on timescales of 5-10 fs [28,29] (for $c-c$ ) and up to $100 \mathrm{fs}$ (for $c$-ph) [30-34]. Despite the plethora of existing investigations on graphite, the exact mechanism behind electronphonon coupling (EPC) and the involvement of the various phonon modes together with their time evolution is unclear. The role of the two prominent (strongly coupled) phonons, the $A_{1}^{\prime}$ and $E_{2 g}$ phonons, their real-time evolution, and relative contribution to EPC have never been measured. Identification of the loss channel and the nature of deexcitation is especially important when considering that EPC is the microscopic origin for $1 / f$ noise [35] in electronic circuits, and the main reason for decoherence in quantum computing [36,37]. Similarly, the scattering mechanisms that photoexcited carriers undergo in bulk graphite are still under debate $[28,38]$ despite the prominence of graphite in devices for energy transfer and storage.

To address these open points for graphite, we first establish the equilibrium (not photoexcited) electronic structure of 95-nm freestanding graphite by measuring the XANES with a 165-as FWHM SXR probe pulse whose spectrum ranges from 250 to $500 \mathrm{eV}$ [39-41]. This SXR probe pulse accesses the $K$-edge core-to-valence transition from the carbon $1 s$ state (bound by $284.2 \mathrm{eV}$ ) and the broad spectral bandwidth of the SXR pulse ensures probing unoccupied electronic states around, and far above, the
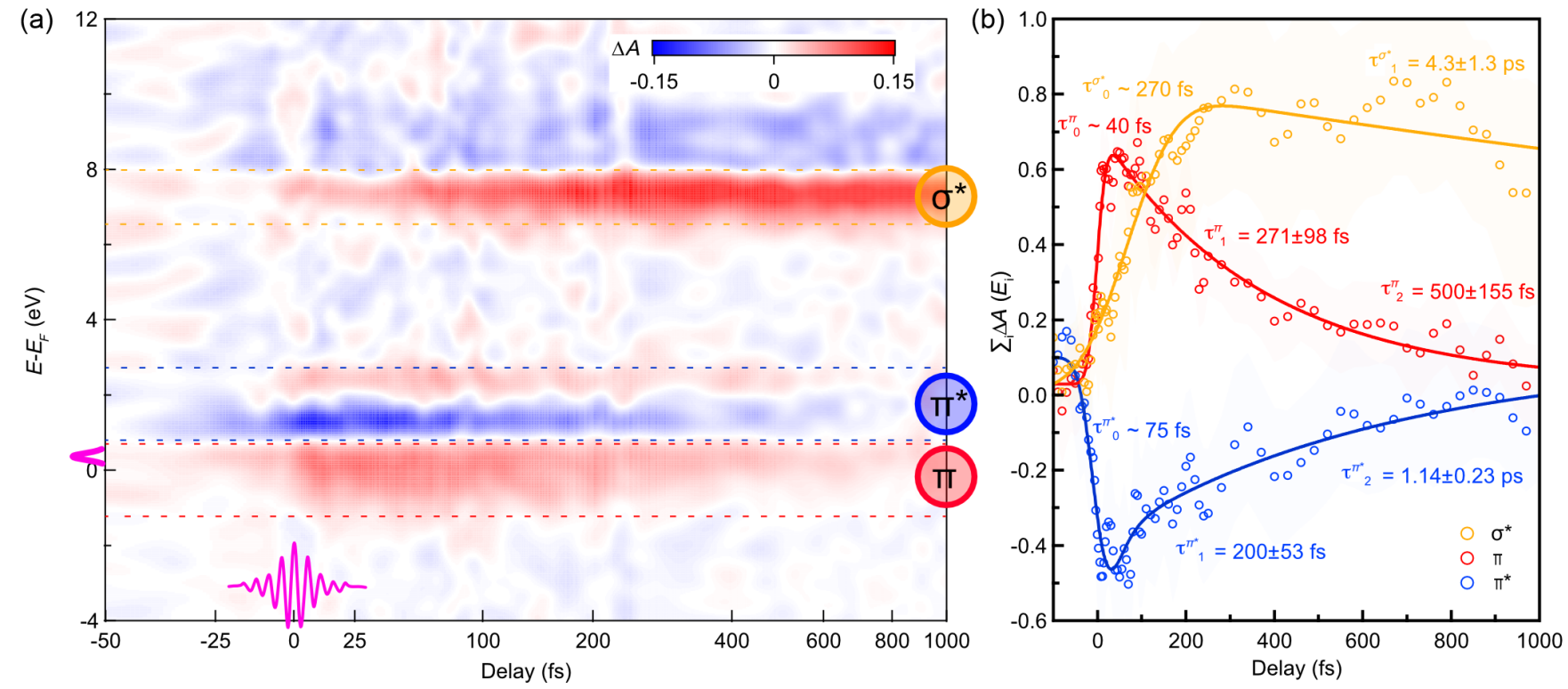

FIG. 2. Time-dependent XANES measurement. (a) Shown is the differential absorption $\Delta A(E)$ between the XANES spectrum with and without pump pulse. The observed features are identified as arising from electrons at the bottom of the CB $\left(\pi^{*}\right)$, holes at the top of the valence band $(\pi)$, and predominately from optical phonons $\left(\sigma^{*}\right)$; note that the material is doped to 650 meV. The pump pulse bandwidth and duration are indicated in pink. (b) Sum of the differential absorption $\sum_{i} \Delta A\left(E_{i}\right)$ over the respective energy range for $\pi$ (red), $\pi^{*}$ (blue), and $\sigma^{*}$ (yellow). The curves are fitted with a double exponential convolved with a Gaussian. 
Fermi level at once. Figure 1(b) shows the calculated band structure together with its orbital character. We orient the material's basal plane at $40^{\circ}$ with respect to the linearly polarized SXR pulse to probe a wide range of orbital character [40]. This allows us to interrogate the $p_{z}$ orbitals, normal to the basal plane, which constitute the $(\pi)$ valence band (VB) and $\left(\pi^{*}\right)$ conduction band (CB). In contrast, $s p_{2^{-}}$ hybridized orbitals $\left(\sigma^{*}\right)$ lie inside the basal plane and are thus very sensitive to in-plane phonon motion. Figure 1(b) (right) shows the excellent agreement between the calculated density of states multiplied by the Fermi-Dirac (FD) function at room temperature with the measured static absorbance, revealing unoccupied electronic states. The $\pi^{*}$ and $\sigma^{*}$ antibonding states, 1.5 and $7 \mathrm{eV}$ above the Fermi energy, are readily identified with the $\bar{M}$ and $\bar{\Gamma}$ points in the Brillouin zone. A Fermi-Dirac fit to the measured absorbance yields that the material is doped with a chemical potential of $650 \pm 45 \mathrm{meV}$.

To study the dynamic flow of energy inside graphite, we photoexcite the material with an ultrashort pump pulse. The pump pulse induces a $\pi-\pi^{*}$ transition at the vicinity of the $\bar{K}$ point [see Fig. 1(b)], thus generating a nonequilibrium condition, which results in the dynamic redistribution of excitation energy across electrons and the lattice. To probe the entire dynamic response, we scan the SXR probe in time from before the pump pulse (negative times, the probe pulse arrives before the pump pulse), across the pump pulse, to after the pump pulse (positive times, the pump pulse arrives before the probe pulse).

The SXR probe pulse is then delayed in time with respect to the excitation (pump) pulse, thus probing via XANES the state of the system at that respective instance in time. To investigate the response of graphite to different excitation conditions, we varied the pump fluence and photon energy for a range of measurements: We used ultrashort $11 \pm 1 \mathrm{fs}$ pump pulses at a photon energy of $0.7 \mathrm{eV}$, and $15 \pm 1 \mathrm{fs}$ at $1.6 \mathrm{eV}$, and varied the pump fluence between $2.8 \pm$ $0.2 \mathrm{~mJ} / \mathrm{cm}^{2}$ and $81 \pm 5 \mathrm{~mJ} / \mathrm{cm}^{2}$ to investigate different regimes of carrier dynamics; see the Supplemental Material (SM) for the complete set of data [11]. Figure 2(a) shows the differential absorption $\Delta A(E)$, which is the difference between the measured absorption of the pumped and of the unpumped material, for the highest fluence of $81 \mathrm{~mJ} / \mathrm{cm}^{2}$ and with $0.7 \mathrm{eV}$ photon energy.

\section{DISCUSSION}

\section{A. Attosecond- to femtosecond-scale dynamics}

Immediately apparent in Fig. 2(a) are changes of up to $15 \%$ in differential absorption, both positive (red) and negative (blue). Figure 1(b) allows us to identify these features as $\pi$ bonding state $(\sim-0.5 \mathrm{eV})$, and as $\pi^{*}(\sim 2 \mathrm{eV})$ and $\sigma^{*}(\sim 7.5 \mathrm{eV})$ antibonding states.

To remove pump-induced broadening and shifting contributions to the line shape, we analyze the absorption spectra at different temporal delays, analog to the procedure detailed in Ref. [42]; see the SM for details [11]. This procedure reveals that the signal between -1.5 and $3 \mathrm{eV}$ originates indeed from occupied (electron) $\pi^{*}$-character CB states and from unoccupied (hole) $\pi$-character VB states. The absorption change between -1.5 and $3 \mathrm{eV}$ is energetically much broader than the bandwidth of the pump pulse, but this is explained by the high carrier concentration of $5 \times 10^{22} \mathrm{~cm}^{-3}$ at the high pump fluence shown in Fig. 2(a). We further find that the signal between 6 and $10 \mathrm{eV}$ originates from a shift and broadening of the $\sigma^{*}$ state at $\sim 7.5 \mathrm{eV}$. Having determined the position of the respective states, we fit a Gaussian function with biexponential decay along their energetic positions. Figure 2(b) shows the result which reveals that the $\pi^{*}$ (electron) signal rises more slowly $\left(\tau_{r}^{e}=75 \pm 5 \mathrm{fs}\right)$ than the $\pi$ (hole) signal $\left(\tau_{r}^{h}=40 \pm 5 \mathrm{fs}\right)$. Conversely, electrons relax faster $\left(\tau_{1}^{e}=200 \pm 53 \mathrm{fs}\right)$ than holes $\left(\tau_{1}^{h}=271 \pm 98 \mathrm{fs}\right)$. Interestingly, all carrier timescales are much longer than the $\mathrm{C} 1 s$ core-hole lifetime in graphite (1.6 fs [43]), which attributes the measured behavior to Debye screening and Landau damping of charge carriers [44]. In contrast, the $\sigma^{*}$ signal exhibits a markedly slower dynamics with a rise time of $\tau_{0}^{\sigma^{*}}=270 \pm 10$ fs and decay time of $\tau_{1}^{\sigma^{*}}=4.3 \pm 1.3 \mathrm{ps}$. This is in accord with expected time evolution of phonon motion.

We now detail the early time dynamics for excitation at a much lower fluence of $2.8 \mathrm{~mJ} / \mathrm{cm}^{2}$ for a photon energy of $1.6 \mathrm{eV}$ and contrast the findings against a similar fluence of $3.2 \mathrm{~mJ} / \mathrm{cm}^{2}$ but with photon energy of $0.7 \mathrm{eV}$; see SM for XANES data [11]. Figures 3(a) and 3(b) show results of the attosecond-resolved measurement with a pump-probe delay step size of 0.6 fs. Immediately apparent in Figs. 3(a) and 3(b) is the buildup of coherent charge oscillations, i.e., polarization of the material. These oscillations occur at occupied states below (orange) and unoccupied states above (blue) the Fermi level. A Fourier analysis reveals that the oscillations occur predominantly at the pump carrier frequency; carrier periods are indicated by the vertical dashed lines in Figs. 3(a) and 3(b). We attribute the observed charge oscillations at the fundamental rather than second harmonic of the optical pump frequency (as observed in attosecond-resolved experiments in non-Dirac materials [45-48]) to the prevalent excursion of carriers in the near-linear potential in close proximity of the $K$ point [49], thus leading to a linear response at the fundamental driving frequency. A low-pass filtered fit through the data (blue and orange solid lines) reveals the incoherent background, which is due to the dephasing of coherent charge oscillation. We observe the concomitant incoherent background rising within a few oscillations of the light field, signifying the ultrafast transfer of energy from the light field into the electron and hole excitation of the material.

An important question that we addressed next is the nature of the observed ultrafast carrier-carrier interaction, during excitation and its dephasing. Our aim is 
Coherent and incoherent dynamics

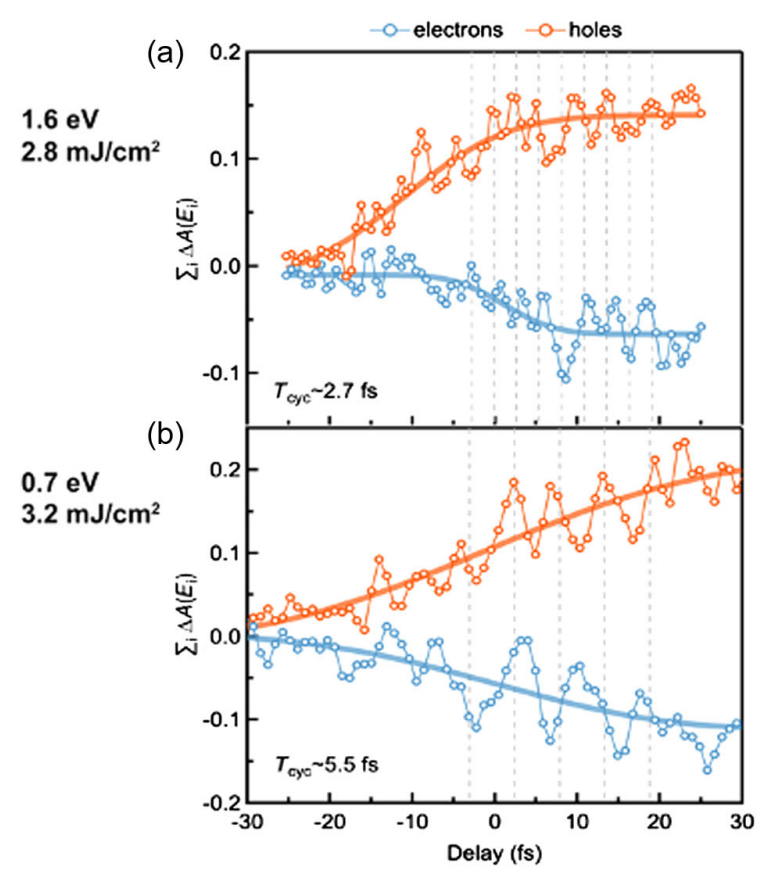

Scattering dynamics

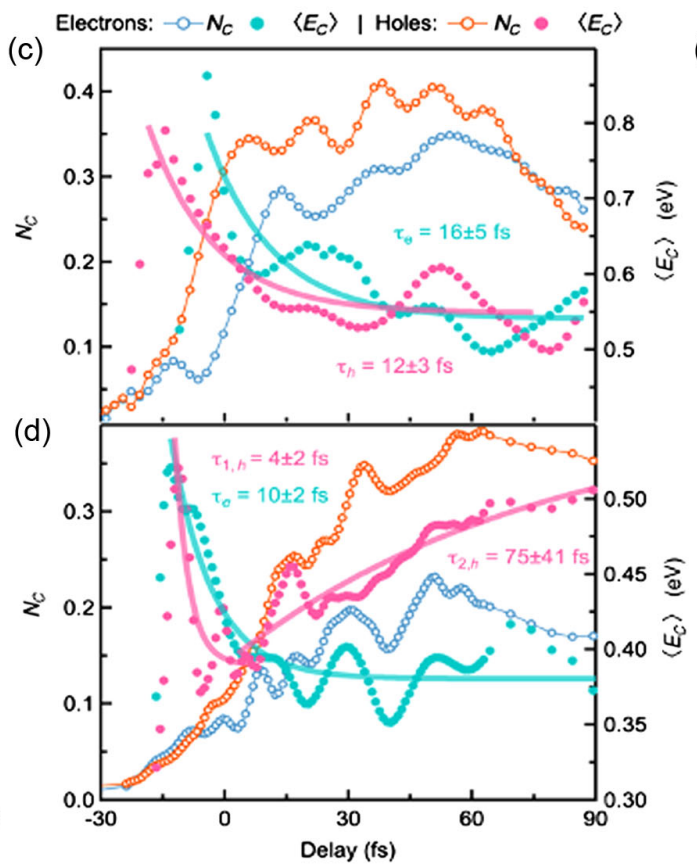

(e)
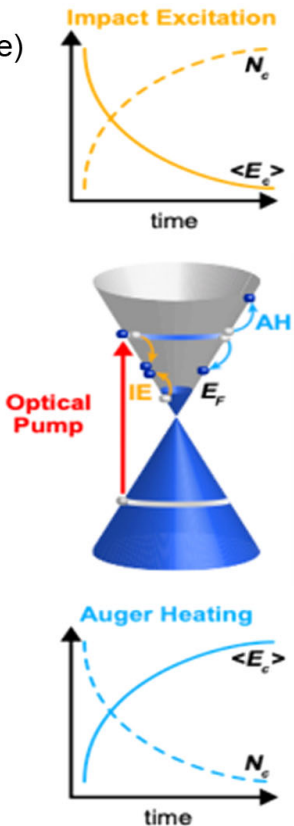

FIG. 3. Carrier dynamics and identification of scattering processes. (a) Sum over the differential absorption $\sum_{i} \Delta A$, which shows the time evolution of electrons (blue) and holes (orange) for a photon energy of $1.6 \mathrm{eV}$ with a fluence of $2.8 \mathrm{~mJ} / \mathrm{cm}^{2}$ and delay step size of $0.6 \mathrm{fs}$. Clearly visible are coherent oscillations at a frequency near the carrier frequency of the light pulse (1.6 eV corresponds to 2.7 fs, marked as dashed vertical lines) and the buildup of the incoherent background leading to material excitation. Panel (b) shows results for a photon energy of $0.7 \mathrm{eV}$ and pump fluence of $3.2 \mathrm{~mJ} / \mathrm{cm}^{2}$. Nicely visible is the buildup of polarization with oscillation frequency of the light carrier wave $(0.7 \mathrm{eV}$ corresponds to $5.5 \mathrm{fs}$, marked as dashed vertical lines) and the material's excitation, albeit at a slower rate. (c),(d) Measurements over a larger temporal range showing the evolution of the number of carriers $N_{c}$ (open symbols) and their average kinetic energy $\left\langle E_{c}\right\rangle$ (closed symbols) for both electrons (blue, petrol blue) and holes (orange, pink). (e) Illustration of the dominant processes, Auger heating $(\mathrm{AH})$, and impact excitation (IE) on the example of graphene. For both processes, we indicate the expected temporal evolution of $N_{c}$ and $\left\langle E_{c}\right\rangle$. (c) The scattering mechanism is IE for both carrier types, electrons and holes. (d) Electrons are dominated by IE while the dynamics of holes is governed by IE at the earliest instances of the pump pulse, but then rapidly dominated by AH.

to distinguish between the pertinent mechanisms of impact excitation and Auger heating [50] and to determine whether both carrier types, electrons and holes, are exposed to identical or different scattering mechanisms. Such an investigation is especially interesting considering that IE leads to a multiplication of carriers as an excited electron (hole) loses energy and momentum in Coulomb scattering, creating an electron-hole pair. AH describes the nonradiative recombination of an electron with a hole where energy and momentum are transferred to an electron (hole) in the same band. Thus, the signature of impact excitation (Auger heating) is an increase (decrease) in the number of carriers with a concurrent decrease (increase) in kinetic energy. Eventually, a dynamic imbalance between electrons and holes will lead to a changing chemical potential and varying probability for carrier recombination. Figure 3(e) depicts how a simple comparison of trends for the two quantities IE and $\mathrm{AH}[51,52]$ allows distinguishing between the mechanisms [50] directly from the measurement. To capture the excitation and the decay of the incoherent background, we conducted additional measurements over a longer time range, up to 90 fs with time steps of 3 fs. Figures 3(c) and 3(d) show the result for which we plot the number of carriers $N_{c}=\sum_{i}\left|\Delta A\left(E_{i}\right)\right|$ together with their occupationnormalized mean (kinetic) energy $\left\langle E_{c}\right\rangle=\sum_{i} E_{i} \Delta A\left(E_{i}\right) /$ $N_{c}$. Examination of the low-fluence $\left(2.8 \mathrm{~mJ} / \mathrm{cm}^{2}\right) 1.6-\mathrm{eV}$ case [Fig. 3(c)] shows a clear signature of IE. In good agreement with Ref. [50], we find from an exponential fit that the number of electrons increases rapidly and thermalizes in $16 \pm 5$ fs. Here, we simultaneously observe the real-time dynamics of Dirac holes, and we find identical behavior, albeit with a slightly faster thermalization time of $12 \pm 3 \mathrm{fs}$.

The power of measuring both carrier types in real time becomes obvious when changing to a pump photon energy of $0.7 \mathrm{eV}$, for similar pump fluence $\left(3.2 \mathrm{~mJ} / \mathrm{cm}^{2}\right)$. Figure $3(\mathrm{~d})$ clearly shows that the measurement of electrons alone would have confirmed IE as a mechanism, while the holes show a more complex behavior. Interestingly, $N_{c}$ increases comparably for electrons and holes up to $70 \mathrm{fs}$. However, $\left\langle E_{c}\right\rangle$ behaves differently: Within $10 \pm 2$ fs ( \pm 2 fs for holes), electrons continue to lose energy while holes initially lose energy but then, on average, gain energy. Holes thus exhibit a 
switchover from IE to AH during the 11-fs duration of the infrared light field. Considering the lower photon energy of $0.7 \mathrm{eV}$, which generates electron-hole pairs much closer to the Fermi energy, the previously inferred $n$ doping of $650 \mathrm{meV}$ leads to an asymmetric phase space for scattering [53]. This asymmetry explains the electron-hole symmetry breaking for scattering in the conduction band and effectively suppresses hole IE [51,54]. To the best of our knowledge, this is the first direct measurement of the real-time dynamics for both carrier types and it shows the importance of such measurement to address problems related to energy storage or light harvesting devices where the recombination of carriers plays a major role in their functionality.

\section{B. Femtosecond- to picosecond-scale dynamics}

Having elucidated the mechanisms of carrier scattering, we now turn to analyzing the full-time range of our measurement, up to $1 \mathrm{ps}$, to investigate electron-phonon scattering and the time evolution of phonons together with their dispersion. Thus, we first fitted the incoherent contribution of the signals shown in Fig. 2(b) to access the coherent dynamics. We employ a three-temperature rateequation model (3TM) [55]. This simple model provides a phenomenological thermodynamic description by subsystems of electrons, strongly coupled optical phonons (SCOPs), and lattice. The transfer of heat from one subsystem to another is described by three coupled differential equations which consider the population averaged kinetics of electrons, SCOPs, and acoustic phonons, and where the coupling strength between subsystems is determined by the heat transfer coefficients. The 3TM fit to the data is shown in Fig. 4(a) and yields that while SCOPs represent only $0.24 \%$ of all vibrational modes, they efficiently absorb $\sim 90 \%$ of the energy from the electronic subsystem due to the large electron-phonon coupling strength of $2.8 \times 10^{16} \mathrm{~W} / \mathrm{m}^{3} \mathrm{~K}$. We note that these values are physically reasonable and in agreement with literature [32].

Next, we investigate the coherent phonon [56,57] signal by analyzing the oscillatory pattern exhibited by the $\sigma^{*}$ data [Fig. 2(b), orange circles, and Fig. 4(b)] with a short-time Fourier transform (STFT) analysis. Such analysis helps in visualizing the phonon dispersion landscape [Fig. 4(d)], and it allows us to identify various dominant coherent phonon modes. We note that any STFT naturally requires balancing frequency versus time resolution: we have thus conducted an additional Fourier analysis of the signal over the entire delay range of 1 ps [Fig. 4(c); see the SM [11]. Figure 4(d) shows that already during and shortly after the laser excitation, coherent motion emerges over a broad range of frequencies. To identify these frequencies, we performed large-scale two-temperature-model molecular dynamics (TTM MD) simulations using an electronictemperature-dependent interatomic potential for graphite (analog to Refs. [58,59]) and the ab initio determined Eliashberg function and $k$-dependent coupling strength; details are given in the Appendix E. Results from the simulation are shown together with experimental data. Figure 4(a) shows the time evolution of the temperatures of electrons, and Fig. 4(b) for SCOPs (the latter calculated directly without approximation from the kinetic energy of the corresponding modes) and the lattice. We find that the match with the experiment is remarkable considering that no fitting parameter was used. Figure 4(e) shows the calculated phonon dispersion for a range of relevant electron temperatures. We determine the two highest phonon frequencies from a multipeak fit to the data and show them in Fig. 4(c) in blue and red. A comparison with the phonon dispersion [Fig. 4(e)] identifies them as the Raman-active $\bar{\Gamma}-E_{2 g}$ and the non-Raman-active $\bar{K}-A_{1}^{\prime}$ SCOPs at $46.4 \pm 2.7$ and $42.7 \pm 1.1 \mathrm{THz}$, respectively. We also note the unexpected observation of very high-frequency coherent lattice oscillations in a forbidden range of the phonon spectrum up to $90 \mathrm{THz}$ [see Fig. 4(c), top left]. These frequencies are commensurate with twice the frequency of the $\bar{K}-A_{1}^{\prime}$ phonon, and, to the best of our knowledge, have not been observed in graphite previously [60]. The capacity to simultaneously measure both the Raman-active $\bar{\Gamma}-E_{2 g}$ and the non-Raman-active $\bar{K}-A_{1}^{\prime}$ SCOP allows us to reveal their temporal dynamics; see the red and blue lineouts across the STFT. Unexpectedly, we find that both SCOPs become dominant already after $20 \mathrm{fs}$ and they reach their maxima at $65.5 \pm 3.0$ and $69.6 \pm 3.0 \mathrm{fs}$, respectively. The early onset of coherent oscillations of the $A_{1}^{\prime}$ mode is startling, considering that the only coherent phonon that can be excited according to present understanding is the Raman-active $E_{2 g}$ mode. Equally surprising, the TTM MD model reveals that the $A_{1}^{\prime}$ SCOP provides the dominant pathway $(\sim 90 \%)$ for deexcitation of the electronic subsystem.

To elucidate the surprising early contribution from the (non-Raman-active) $A_{1}^{\prime}$ mode, we calculated the equilibrium and the laser-excited potential energy surfaces along the $A_{1}^{\prime}$ mode (see SM [11]), and we found no displacement of the potential minimum. Thus, without the possibility for an immediate displacive excitation of the fully symmetric mode, we conclude that the observed coherent lattice motion must originate from the very strong electronSCOPs coupling, thus acting almost impulsively. Our model further explains the unexpected observation of the high-frequency oscillations up to $90 \mathrm{THz}$ at twice the frequency of the $A_{1}^{\prime}$ mode. We find that the DOS is modulated due to the absolute value of the displacement but not its direction, thus resulting in twice the phonon oscillation frequency in the DOS. The simulations also explain the surprising finding that the oscillations of the $A_{1}^{\prime}$ mode are stronger than those of the $E_{2 g}$ phonon, and that it reaches its maximum earlier: The form of the Eliashberg function yields much stronger electron-phonon coupling for the $A_{1}^{\prime}$ mode than for the $E_{2 g}$ mode [59]. Thus, in accord with the experiment, even though the $E_{2 g}$ is impulsively 

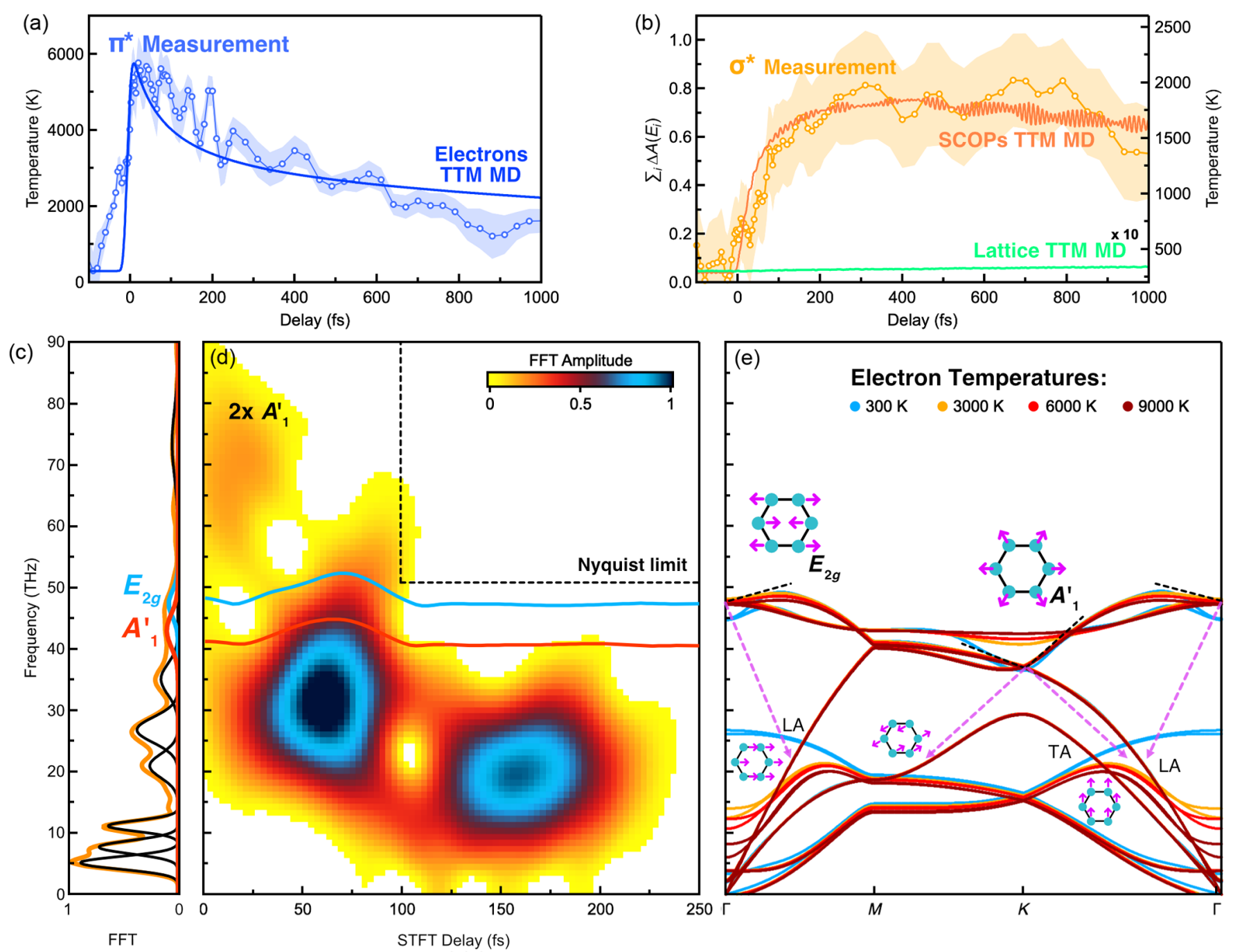

FIG. 4. Phonon dynamics retrieved from attosecond XANES. Panels (a) and (b) show results from the TTM MD simulation (solid lines) together with experimental data from Fig. 2. The evolution of the electronic temperature is shown in (a) while (b) shows the SCOPs and lattice subsystems. Panel (c) shows results from a Fourier analysis of the experimental $\sigma^{*}$ data [shown in (b)]. A multipeak fit reveals the predominant modes; positions of the $A_{1}^{\prime}$ and $E_{2 g}$ are shown in red and blues, respectively. (d) Short-time Fourier transform (STFT) of the $\sigma^{*}$ data (b) over a time range of $250 \mathrm{fs}$, visualizing coherent phonon contributions. A comparison with the calculated phonon dispersion (e), for different electronic temperatures (see SM [11]), allows us to identify the phonon modes, their main branches (dashed lines), and their dispersion into longitudinal (LA) and transvers acoustic (TA) modes (dashed arrows). Striking are the highfrequency oscillations at twice the phonon frequency of $A_{1}^{\prime}$, the simultaneous and fast rise of the coherent Raman active $E_{2 g}$ mode, concomitantly with the non-Raman-active $A_{1}^{\prime}$ mode, and the rapid decay into coherent low-frequency phonons and their temporal dynamics.

excited by the light field via Raman scattering, the main loss channel for electronic deexcitation is via the $A_{1}^{\prime}$ mode due to the very strong EPC. This also explains the striking observation that the coherent oscillation amplitudes of both SCOPs reach their maxima $\sim 60$ fs after the pump pulse. Further analysis reveals excitation of the $\bar{K}-E^{\prime}$ mode at $33.7 \pm 0.1 \mathrm{THz}$, and the observation of lower energetic phonon modes at $26.6 \pm 0.1,19.8 \pm 0.1$, and $16.5 \pm$ $0.1 \mathrm{THz}$, which peak after $\sim 160 \mathrm{fs}$. This observation is in agreement with recent results by Stern et al. [61] from ultrafast electron diffraction, and suggestive of parametric difference frequency generation $[62,63]$ from $E_{2 g}$ and $A_{1}^{\prime}$ phonons into less energetic coherent transverse and longitudinal acoustic phonon modes.

\section{CONCLUSIONS}

In conclusion, we demonstrate the ability to track energy flow upon light absorption between electrons, holes, and phonons in real time. We applied the method to graphite and demonstrate its capability by disentangling the coherent and incoherent excitation and dissipation pathways 
from the attosecond to the picosecond. At earliest times, the dynamics of electrons and holes reveal disparate scattering mechanisms for infrared excitation, close to the Fermi energy. We find that impact excitation governs ultrafast dephasing of the coherent carrier dynamics for electrons. At the same time, holes exhibit a switchover from impact excitation to Auger heating during the 11-fs duration of the infrared light field. We attribute this switchover to the limited scattering phase space in the $n$-doped material.

Further, the measurement sheds new light on longstanding questions regarding the excitation of SCOPs in graphite. We directly observe the non-Raman-active $A_{1}^{\prime}$ phonon concomitantly with the Raman-active $E_{2 g}$ phonon, and we find that both SCOPs are coherently excited within $20 \mathrm{fs}$ of the pump pulse. The coherent excitation of both SCOPs is nondisplacive and is explained by the strong electron-phonon scattering, i.e., via a seemingly incoherent process. We identify the $A_{1}^{\prime}$ phonon as the dominating channel for the dissipation of electronic coherence. Moreover, unobserved in graphite, we find high-frequency oscillations up to $90 \mathrm{THz}$, which arise from the modulation of the electronic DOS by the atomic displacements along the $E_{2 g}$ and $A_{1}^{\prime}$ modes.

These measurements establish the utility of attosecond core-level XANES spectroscopy to achieve a detailed and simultaneous view on the temporal evolution of the photoncarrier-phonon system with surprising new results even for a seemingly well-studied system like graphite. The method is generally applicable to molecules, liquids, and solids, with the only requirement that the attosecond pulse spectrum covers an elemental absorption edge of the system under investigation.

We emphasize that attosecond core-level XANES is different from general attosecond absorption spectroscopy as it combines attosecond resolution with the unambiguous electronic-state mapping of $K$-shell XANES spectroscopy. Attosecond pulses are ideal for XANES since their extremely large spectral bandwidth presents no impediment on the energy resolution of the measurement. Further, using a carrier-envelope phase-controlled pump pulse even reduces the temporal resolution to (essentially) the duration of the attosecond probe pulse itself.

The ongoing development of $\mathrm{x}$-ray light sources, either high-harmonic-based or free-electron lasers, will bring the methodology to elemental core-level edges of heavier elements in the hard $\mathrm{x}$-ray spectral range. Very exciting are recent developments on $\mathrm{x}$-ray gratings and detector technology. The availability of high-efficiency and highresolution SXR zone plates, in combination with direct $\mathrm{X}$-ray detection, provides an improvement by more than one order of magnitude in sensitivity and resolution. These improvements are very significant as they reduce measurement time to a mere tens of minutes and they will provide sufficient spectral resolution to discriminate detailed chemical shifts and vibrational signatures which are presently not resolvable. Equally exciting are recent theoretical developments that aim at describing x-ray absorption as nonlinear two-photon interaction, thus addressing important aspects such as dynamic core-hole effects or exciton-phonon coupling. We thus expect that the general applicability of the method will prove valuable to address questions such as, for instance, the energy dissipation in light harvesting, organic electronic and energy storage systems, or to reexamine long-standing questions in nonequilibrium multibody physics such as phase transitions and superconductivity.

\section{ACKNOWLEDGMENTS}

J. B. acknowledges financial support from the European Research Council for ERC Advanced Grant "TRANSFORMER" (788218), ERC Proof of Concept Grant "miniX" (840010), FET-OPEN "PETACom" (829153), FET-OPEN “OPTOlogic" (899794), LaserlabEurope (871124), Marie Skłodowska-Curie ITN "smart-X" (860553), MINECO for Plan Nacional PID2020-112664 GB-I00; AGAUR for 2017 SGR 1639, MINECO for "Severo Ochoa" (SEV- 2015-0522), Fundació Cellex Barcelona, the CERCA Programme/Generalitat de Catalunya, and the Alexander von Humboldt Foundation for the Friedrich Wilhelm Bessel Prize. M. R. acknowledges Marie Skłodowska-Curie Grant Agreement No. 754510 (PROBIST). S. Sharma thanks DFG for funding through TRR227 (Project No. A04). M. E. G. acknowledges support from DFG through Grant No. GA465/15-2. The large-scale MD simulations were performed on the Lichtenberg High Performance Computer (HHLR) at the TU Darmstadt. We thank J. Menino for his technical support.

J. B. conceived and supervised the project. N. D. P., T.P.H.S., and D.E.R. performed the experiments with support from S. Severino and M. R., T. P. H. S., and N. D. P. analyzed the data with support from J. B. and D. E. R. B. N. contributed to the experimental setup. T. V. developed the 3TM model. J. B. performed electronic structure calculations. P. E., K. D., and S. S. performed the RT-TDDFT calculation with Ehrenfest dynamics, M. Uemoto and $\mathrm{K}$. Yabana performed TD-DFT calculations on conductivity, B. B., S. K., and M. E. G. performed TTM-MD and DFT simulations. Y. J. provided X-ray absorption calculations. T.D. and C.R. provided the graphite samples. R.E., M.E. G., and M. W. contributed to the data interpretation. J. B., T. P. H. S., N. D. P., and D. E. R. wrote the manuscript with contribution from M.E. G. and R. E.

Note added in proof.-Recently, we became aware of a theory paper which further supports the importance of the hot phonon dynamics on an ultrafast timescale [64]. 


\section{APPENDIX A: EXPERIMENTAL SETUP}

X-ray absorption near edge structure spectroscopy measurements are performed in a homebuilt soft X-ray beam line in which a Mach-Zehnder interferometer permits attosecond-resolved pump-probe experiments; see Fig. 1 in SM for a sketch of the setup [11]. A 380- $\mu \mathrm{J}, 1850-\mathrm{nm}$ $(0.7 \mathrm{eV})$, sub-2-cycle, carrier-envelope phase-stable pulse from a homebuilt 1-kHz laser system [65] is sent into the beam line where it enters the Mach-Zehnder interferometer. Depending on the experiment, this pulse is split by a $95 / 5$ (low-fluence case) or 70/30 (high-fluence case) beam splitter and the low-energy portion is directed as pump to the target. The high-energy part is focused into a highpressure gas target [66-68], with backing pressures up to 12 bar in helium. The condition for the present experiment was identical to the ones in Ref. [68] for producing an isolated 165-as pulse in the SXR water window [41] with a spectrum from 250 to $500 \mathrm{eV}$. The emerging SXR pulse is sent through a 100-nm Sn filter to reject leftover infrared radiation before being focused onto target with a SXR ellipsoid (Carl Zeiss AG) to a target beam size of $15 \pm$ $5 \mu \mathrm{m}$ FWHM. After the target, a second 100-nm Sn filter is used to reject the pump light before the SXR radiation is dispersed with a homebuilt SXR spectrograph, which consists of a flat-field aberration-corrected and reflecting concave grating (2400 lines/mm; Hitachi High Technologies America, Inc.) and a cooled, back-illuminated CCD (PIXIS$\mathrm{XO}$; Princeton Instruments); the resolution of the spectrograph is $0.3 \mathrm{eV}$ at the carbon $K$ edge at $284 \mathrm{eV}$. The pump-probe delay is controlled via a closed-loop delay stage (Smaract $\mathrm{GmbH}$ ) and the pump beam intersects the target at $2^{\circ}$ with respect to the SXR beam. The pump beam focuses to $50 \pm 1 \mu \mathrm{m}$ FWHM, which is almost 3 times larger than the probe spot size, which ensures probing a homogeneously pumped region of the sample by the much smaller SXR beam. The basal plane of $95-\mathrm{nm}$ freestanding graphite is oriented at $40^{\circ}$ with respect to the linearly $p$-polarized SXR pulse for the selective probing of bands with different orbital character, here predominantly the $\bar{\Gamma}-\bar{K}-\bar{M}-\bar{\Gamma}$ directions and the pump is polarized to maximize the optical excitation from $\pi$ to $\pi^{*}$ band near the $\bar{K}$ point of the band structure of graphite. For the experiments with the 800-nm (1.6-eV) pump beam, a frequency-doubling stage is inserted on the pump beam path, consisting of a $200-\mu \mathrm{m}$-thick BBO crystal and a pair of dichroic mirrors to separate the second harmonic from the fundamental. Four reflections from chirped mirrors (Layertec $\mathrm{GmbH}$ ) recompress the 800-nm pulse to $15 \pm 1 \mathrm{fs}$ (see Fig. 2 in SM [11]); the quoted pump pulse durations were retrieved at the SXR target plane from second-harmonic frequency-resolved optical gating (FROG) measurements.

\section{APPENDIX B: DATA ACQUISITION}

The data acquisition protocol consists of a collection of series of spectra with different combinations of pump and probe beams on the sample. Shutters along the probe and the pump path are synchronized with the CCD camera and were used to alternate in a sequence of 15 spectra of 40 -s integration for the pump plus probe $\left(\tau_{p p}\right), 15$ spectra of 40-s integration for the probe $\left(\tau_{0}\right)$, and 4 spectra of $40 \mathrm{~s}$ for the pump $\left(\tau_{p}\right)$ case. This scheme allows us to minimize the influence of possible SXR spectral fluctuations on the analysis, while the pump-only acquisition enables the correct background subtraction. Comparing the probe-only spectra for a constant delay no effects of sample heating could be observed in the spectra. Each delay time step between pump and probe resulted in 25 min of measurement time.

For each of the acquisitions, we record the raw CCD image in a predefined region of interest (ROI) which was identical for $\tau_{p p}, \tau_{0}$, and $\tau_{p}$. Each ROI is then summed together to obtain the spectrum for each delay step sequence $\tau_{p p}, \tau_{0}$, and $\tau_{p}$. The detector's dark and thermal noise are removed from each spectrum. To remove the possible residual pump background the $1 \mathrm{D} \tau_{p}$ spectrum is multiplied by $15 / 4$ to account for the reduced integration time, then subtracted from $\tau_{p p}$ spectrum. To account for possible slow drifts in the SXR flux, despite the 40-s integration windows, we normalize to the energy range between 200 and $280 \mathrm{eV}$ before carbon $K$ edge for $\tau_{p p}, \tau_{0}$, and $\tau_{p}$, and we apply a three-pixel boxcar average. These traces are taken to calculate the differential transmission $\left(\Delta T=T_{p p}-T_{0}\right)$ normalized to the probe-only spectrum, $\Delta T / T_{0}$.

From the differential transmission spectrum, we can obtain the change of absorption $(\Delta A)$ through $-\ln [(\Delta T /$ $\left.\left.T_{0}\right)+1\right]=\Delta A$. We used the identity $\left(\Delta T / T_{0}\right)=$ $\left(I_{0} e^{-\alpha_{p p} d}-I_{0} e^{-\alpha_{0} d} / I_{0} e^{-\alpha_{0} d}\right)=e^{-\Delta \alpha d}-1$, with the attenuation coefficient $\alpha$ and sample thickness $d$.

\section{APPENDIX C: CARRIER MULTIPLICATION- IMPACT EXCITATION VERSUS AUGER HEATING}

The two carrier multiplication processes, impact excitation and Auger heating occur on similar timescales after photoexcitation [69]. To highlight their strong dependency on pump fluence and carrier concentration, we followed the approach presented by Gierz et al. [50], and distinguish the two processes by the scaling of normalized average kinetic energy $E_{c}$ and number of carriers $N_{c}$.

XANES probes unoccupied electronic states near the Fermi level $n_{u c}[70,71]$ and thus the measured spectrum is proportional to the unoccupied density of states:

$$
a(E) \propto n_{u c}(E) \propto[1-\mathrm{FD}(E)] \times \operatorname{DOS}(E) .
$$

Here, the Fermi-Dirac distribution accounts for the electronic occupation in thermodynamic equilibrium at temperature $T_{e}$ and with Fermi energy $E_{F}$. 
TABLE I. Carrier multiplication factor.

\begin{tabular}{lcccc}
\hline \hline Fluence & $\Delta A_{e}\left(\Delta A_{h}\right)$ & $n_{\text {opt }}\left(\mathrm{cm}^{-3}\right)$ & $n_{f}^{e}\left(n_{f}^{h}\right)\left(\mathrm{cm}^{-3}\right)$ & $\mathrm{CM}_{e}\left(\mathrm{CM}_{h}\right)$ \\
\hline High $[1.85 \mu \mathrm{m}]$ & $0.3(0.45)$ & $4.83 \times 10^{22}$ & $7.20 \times 10^{21}\left(6.75 \times 10^{21}\right)$ & $0.15(0.14)$ \\
Low $[1.85 \mu \mathrm{m}]$ & $0.1(0.2)$ & $1.86 \times 10^{21}$ & $2.40 \times 10^{21}\left(3.00 \times 10^{21}\right)$ & $1.29(1.61)$ \\
Low $[0.8 \mu \mathrm{m}]$ & $0.15(0.2)$ & $8.6 \times 10^{20}$ & $3.60 \times 10^{21}\left(3.00 \times 10^{21}\right)$ & $4.19(3.49)$ \\
\hline \hline
\end{tabular}

Changes in the carrier occupation are evident from $\Delta A$, the difference between the pumped and unpumped absorption spectrum. The sum over all the apparent occupation changes $\Sigma_{i}\left|\Delta A\left(E_{i}\right)\right|$ then highlights the number of excited carriers $N_{c}$ across the probed sample volume:

$$
\begin{aligned}
\Sigma_{i} A\left(E_{i}\right) & \propto \Sigma_{i}\left[1-\mathrm{FD}\left(E_{i}\right)\right] \times \operatorname{DOS}\left(E_{i}\right) \propto N_{u c} \\
& \rightarrow \Sigma_{i}\left|\Delta A\left(E_{i}\right)\right| \propto N_{c} .
\end{aligned}
$$

Note that the energy region in the DOS, where changes in the soft X-ray absorption appear is dominated by $\pi^{*}$ states, which relate to electronics states near the $\bar{K}$ and $\bar{H}$ points. The near-infrared pump pulse, however, only excites carriers near $\bar{K}$.

The average kinetic energy that is the energy corresponding to the center of mass of the carrier distribution is calculated from the measurement, for both carrier types, electrons and holes,

$$
\left\langle E_{c}\right\rangle=\frac{\Sigma_{i}\left(E_{i}\right) \Delta A\left(E_{i}\right)}{\Sigma_{i}\left|\Delta A\left(E_{i}\right)\right|},
$$

where we use the energy $E_{i}$ referenced to the Fermi energy. This approach allows the identification of the various contributions to (hot) carrier multiplication for electrons and holes; see Fig. 3 in the main text.

To quantify carrier multiplication, we calculate a carrier multiplication factor CM, in analogy to the work of Winzer and Malić [54],

$$
\mathrm{CM}=\frac{n_{f}-n\left(T_{0}\right)}{n_{\mathrm{opt}}},
$$

with the final carrier density $n_{f}$, the optically excited carrier density $n_{\mathrm{opt}}$, and the initial thermal carrier density $n\left(T_{0}\right)$. The difference between final and initial carrier density is given by the measured absorption changes $\Delta A$ times the density of states over the apparent energy region. We integrate the calculated DOS over the energy range of the signal to obtain $n=2.4 \times 10^{22}$ states $/ \mathrm{cm}^{3}$ $\left(1.5 \times 10^{22}\right.$ states $\left./ \mathrm{cm}^{3}\right)$ for electrons (holes) [0.848 (0.539) states/unit cell; $\left.V_{\text {unit cell }}=35 \times 10^{-24} \mathrm{~cm}^{3}\right]$. Lastly, we can calculate the carrier multiplication factor as

$$
\mathrm{CM}=\frac{\Delta A n}{\alpha I / \hbar \omega D} .
$$

In Table I we present the calculated CM for the three measurements for electrons and holes.

\section{APPENDIX D: THREE-TEMPERATURE MODEL}

To quantify the incoherent contribution, we resort to a thermodynamic description between electrons, strongly coupled optical phonons, and lattice with a three-temperature rate-equation model [55] fit to the data.

The heat capacity of the electrons at temperature $T_{E}$ is given by

$$
C_{E}=\gamma T_{E},
$$

with $\gamma=2.5 \mathrm{~J} / \mathrm{m}^{3} \mathrm{~K}^{2}$ based on literature values [72]. In the model, the SCOPs have an energy of $\hbar \omega=200 \mathrm{meV}$ and their heat capacity is calculated according to the formula [16]

$$
C_{\mathrm{SC}}=\frac{d}{d T} \frac{f N \hbar \omega}{\exp \left(\hbar \omega / k_{B} T\right)-1},
$$

where $N=3.4 \times 10^{29} \mathrm{~m}^{-3}$ is the total number of vibrational modes per volume for graphite and $f$ is the fraction of these modes that are SCOPs. Finally, the heat capacity of the lattice $\left(C_{L}\right)$ is found from the work of Pop et al. [73]. The equations of the $3 \mathrm{TM}$ are used for a nonlinear fitting of the experimental data, where the fitted parameters are the coupling constants $\left(G_{E-\mathrm{SC}}\right.$ and $\left.G_{\mathrm{ph}-\mathrm{ph}}\right)$, the fraction of SCOPs $(f)$, and the incident laser fluence.

Based on the fitting, SCOPs represent $0.24 \%$ of all the vibrational modes, they are excited by hot electrons with an energy transfer rate $G_{E-\mathrm{SC}}=2.8 \times 10^{16} \mathrm{~W} / \mathrm{m}^{3} \mathrm{~K}$, and they cool down through phonon-phonon coupling with an energy transfer rate $G_{\mathrm{ph}-\mathrm{ph}}=5.5 \times 10^{16} \mathrm{~W} / \mathrm{m}^{3} \mathrm{~K}$. Fitting the modeled temperature evolution with a biexponential decay shows that the time constants for heating and cooling of SCOPs are $\tau_{1}=120 \mathrm{fs}$ and $\tau_{2}=600 \mathrm{fs}$, respectively. We find that the strong electron energy transfer to SCOPs dominates the incoherent signal until 500 fs before phononphonon scattering becomes increasingly dominant.

\section{APPENDIX E: MOLECULAR DYNAMIC SIMULATION $e$-ph OF DYNAMICS}

For simulating graphite's electron and phonon dynamics, shown in Fig. 5, we utilized a two-temperature model molecular dynamics [74] scheme considering the electron- 

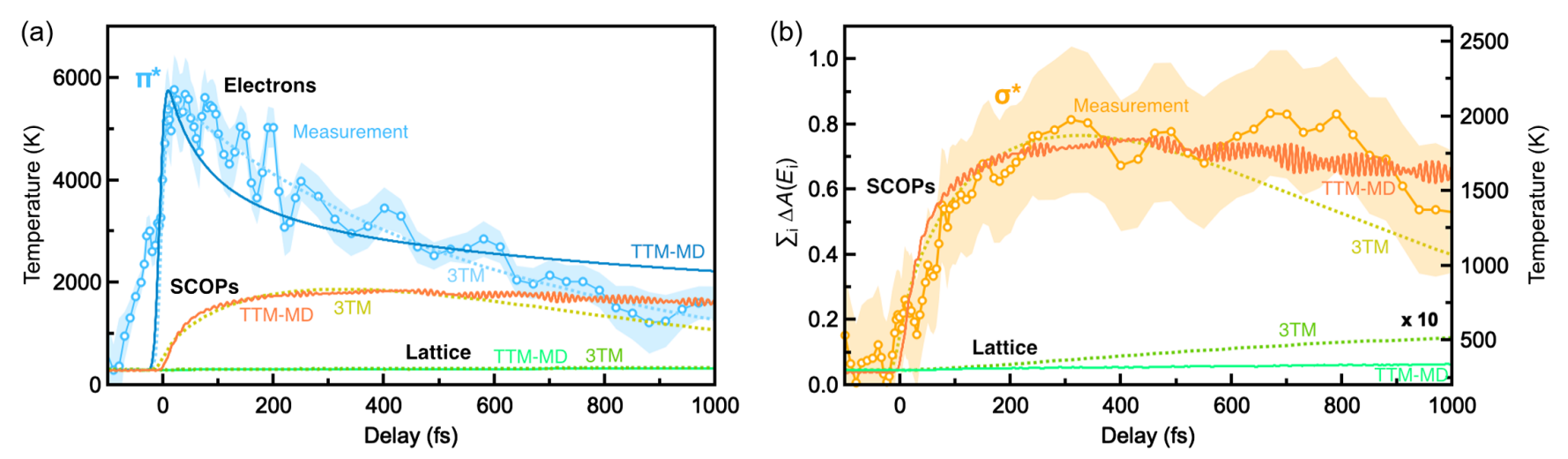

FIG. 5. (a) Temporal evolution of the temperature of the electronic system from a Fermi-Dirac (FD) fit to the experimental data (blue, open circles). The three-temperature model (3TM) fit reproduces the electronic temperature evolution (blue, dotted line) and provides the evolution of the SCOPs (yellow, dotted line) and lattice (dark green, dotted line) subsystems. Overlaid are also results from the TTM MD simulation (solid lines) for all three subsystems. (b) Comparison between the $\sigma^{*}$ experimental signal (yellow, open circles), with SCOPs (red), and lattice (brown) temperatures obtained from the 3TM model in (a). Overlaid is also the result from the TTM MD simulation, confirming the phononic origin of the $\sigma^{*}$ signal. Clearly visible are high-frequency oscillations in the SCOPs which are predicted by the TTM MD model (dark orange, solid line) which are in agreement with the experimental result shown in Fig. 4(a).

phonon coupling only to selected phonon modes-the SCOPs-as described in Ref. [59]. But in contrast to Ref. [59], we employed an electronic-temperaturedependent interatomic potential, which we developed from $a b$ initio reference MD simulations using the method described in detail in Ref. [75]. We performed these reference simulations on laser-excited potential energy surfaces with the density functional theory molecular dynamics codes CHIVES (code for highly excited valence electron systems) [76]. The exchange-correlation part of the energy functional is approximated by the local density approximation [77]. CHIVES uses atom-centered Gaussian basis sets [78], relativistic pseudopotentials [79,80], and periodic boundary conditions. For the reference simulations, we used a supercell containing a graphite film with 252 atoms and a supercell containing graphene with 180 atoms. The finally obtained interatomic potential contains 31 coefficients and has a cutoff radius of $0.34 \mathrm{~nm}$. The coefficients are polynomials of order 4 with respect to the electronic temperature. We verified the description of the phonon band structure, the electronic specific heat, and the interatomic forces and energies for graphene and graphite. For the simulations with the electronictemperature-dependent interatomic potential, we have generated a graphite supercell containing 16128 atoms. The supercell was chosen in such a way that it contains the $K$ and $K^{\prime}$ point, which are important for the electron-phonon coupling. The supercell was thermalized by initializing the velocities and performing a thermalization run. This procedure is repeated until the supercell obtains a temperature of about $300 \mathrm{~K}$. The laser excitation of graphite is simulated by increasing the electronic temperature according to a Gaussian-shaped pulse of a FWHM time width of $15 \mathrm{fs}$, where every atom absorbs $0.06 \mathrm{eV}$ on average. The electronic temperature modifies the Fermi-Dirac function, which is assumed for occupying the electronic states. This procedure is justified because graphite reaches a well-defined electronic temperature within about 30-50 fs after irradiation [81-85]. Graphite exhibits a particularly strong electron-phonon coupling, which is modeled as for graphene in Ref. [59]. The same Eliashberg function, $k$-dependent coupling strength integrated electron-phonon coupling constants as for graphene are used, multiplied by the number of graphene planes in the supercell. In total, 980 phonon modes were identified as SCOPS in our simulation cell and were used for considering the electron-phonon coupling. The electron-phonon coupling constant was derived from $a b$ initio based on the method developed by Allen [86]. More details can be found in Ref. [59].

Figure 4(e) of the main text shows the phonon dispersion of graphite from CHIVES after ultrafast laser excitation with electronic temperatures of 300,3000,6000, and $9000 \mathrm{~K}$, respectively. During these calculations the atoms are in their equilibrium positions. Figure 4(d) shows that the laser excitation strongly modifies the interaction between the atoms. In particular, the optical out-of-plane phonon modes at the $\Gamma$ point are strongly softened due to the laser pulse. The optical in-plane phonon modes, on the other hand, exhibit a bond hardening near the $\Gamma$ point and $K$ point.

The velocity autocorrelation function (VAC) gives a measure for the strength of interactions in the material. It is calculated by

$$
\operatorname{VAC}(\tau, T)=\int_{0}^{T} d t \frac{1}{N} \sum_{i=1}^{N}\left[v_{i}(t) v_{i}(t+\tau)\right]
$$

where $N$ is the number of atoms, and $v_{i}(t)$ the velocity of atom $i$. The calculated VAC depends on the time shift $\tau$ and 
the integrating time $T$. The VAC after laser excitation is shown in Fig. 12(a) of the Supplemental Material for time shifts $\tau$ from 0 to $250 \mathrm{fs}$ and integrating $T=100 \mathrm{fs}$ [11]. The VAC shows clear laser-induced oscillations between 40 and $50 \mathrm{THz}$ close to the SCOPs frequencies and their parametric equivalent.

\section{APPENDIX F: REAL-TIME TDDFT SIMULATION OF CARRIER OCCUPATION AND IMPRINT OF NUCLEAR MOTION}

To study the early field driven electron-phonon dynamics in graphite we apply a parameter-free $a b$ initio method; time-dependent density functional theory (TDDFT) [87]. A regular mesh in $\mathbf{k}$ space of $24 \times 24 \times 10$ was used and a time step of $\Delta t=4.13 \mathrm{fs}$ was employed for the time-propagation algorithm [88]. A smearing width of $0.027 \mathrm{eV}$ was used. The laser pulse used in the simulations is linearly polarized with polarization vector in plane and has a $13 \mathrm{fs}$ (FWHM) pulse length, $800 \mathrm{~nm}$ wavelength ( $1.55 \mathrm{eV}$ photon energy), and an incident fluence of $F=9.6 \mathrm{~mJ} / \mathrm{cm}^{2}$. All calculations are performed using the ELK code [89],] which uses the full potential linearized augmented-plane-wave method. The time-dependent Kohn-Sham orbitals as two-component Pauli spinors are as follows:

$$
i \frac{\partial \psi_{j}}{\partial t}=\left[\frac{1}{2}\left(-i \boldsymbol{\nabla}+\frac{1}{c} \boldsymbol{A}_{\mathrm{ext}}(t)\right)^{2}+v_{s}(\boldsymbol{r}, t)+\frac{1}{2 c} \boldsymbol{\sigma} \cdot \boldsymbol{B}_{s}(\boldsymbol{r}, t)+\frac{1}{4 c^{2}} \boldsymbol{\sigma} \cdot\left(\boldsymbol{\nabla} v_{s}(\boldsymbol{r}, t) \times-i \boldsymbol{\nabla}\right)\right] \psi_{j}(\boldsymbol{r}, t),
$$

with $\sigma$ referring to the Pauli matrices. The Kohn-Sham effective potential $v_{s}(\boldsymbol{r}, t)=v_{\text {ext }}(\boldsymbol{r}, t)+v_{H}(\boldsymbol{r}, t)+v_{\mathrm{xc}}(\boldsymbol{r}, t)$ consists of the external potential $v_{\text {ext }}$, the classical electrostatic Hartree potential $v_{H}$, and the exchange-correlation (XC) potential $v_{\mathrm{rm}}$, while the Kohn-Sham magnetic field is $\boldsymbol{B}_{s}(\boldsymbol{r}, t)=\boldsymbol{B}_{\mathrm{ext}}+\boldsymbol{B}_{\mathrm{xc}}(\boldsymbol{r}, t), \boldsymbol{B}_{\mathrm{ext}}(\boldsymbol{r}, t)$ being the magnetic field of the laser pulse and $\boldsymbol{B}_{\mathrm{xc}}(\boldsymbol{r}, t)$ the XC magnetic field. We use the adiabatic local spin density approximation for $\boldsymbol{B}_{\mathrm{xc}}(\boldsymbol{r}, t)$; see Krieger et al. [90]. The last term in the equation represents spin-orbit coupling. $\boldsymbol{A}_{\text {ext }}(t)$ is the vector potential representing the incident pump field. We solve the above equation for the electronic system alone.

Coupled dynamics of the electron-nuclear system is then included at the Ehrenfest level of approximation in which the atomic forces are calculated at each electronic time step and the atomic positions are updated according to Newton's equation of motion. It must be stated that our implementation is valid for small amplitude motion only. Two complete runs of the time evolution are performed: the atomic forces are calculated during the first and the effects of the backreaction of the atomic displacements are included in the second. The muffin tins themselves are not displaced because this would change the augmented plane wave basis itself and require that the time-evolving Kohn-Sham states be reexpanded in the new basis.

Instead, the linear-order change in the Coulomb potential (nuclear plus Hartree from core density) given by

$$
\delta V_{\mathrm{Cl}}(\boldsymbol{r}, t)=-\sum_{\alpha} \delta \boldsymbol{u}_{\alpha}(t) \cdot \nabla_{\boldsymbol{u}_{\alpha}} V_{\mathrm{Cl}}(\boldsymbol{r}),
$$

where $\delta \mathbf{u}_{\alpha}(t)$ is the atomic displacement for atom $\alpha$, is added to the time-dependent Hamiltonian. A further limitation of the augmented plane wave basis means that this potential will be underscreened leading to a possible overestimation of the effects of the nuclear motion.
[1] L. D. Landau and E. M. Lifshitz, The Classical Theory of Fields (Butterworth-Heinemann, London, 1975).

[2] C. Chen et al., Distinguishing Attosecond ElectronElectron Scattering and Screening in Transition Metals, Proc. Natl. Acad. Sci. U.S.A. 114, E5300 (2017).

[3] K. Barmak and K. Coffey, Metallic Films for Electronic, Optical, and Magnetic Applications ScienceDirect (Woodhead Publishing, Sawston, United Kingdom, 2014).

[4] S. R. Forrest, Excitons and the Lifetime of Organic Semiconductor Devices, Phil. Trans. R. Soc. A 373, 1 (2015).

[5] A. Classen, C. L. Chochos, L. Lüer, V. G. Gregoriou, J. Wortmann, A. Osvet, K. Forberich, I. McCulloch, T. Heumüller, and C. J. Brabec, The Role of Exciton Lifetime for Charge Generation in Organic Solar Cells at Negligible Energy-Level Offsets, Nat. Energy 5, 711 (2020).

[6] J. Bardeen, L. N. Cooper, and J. R. Schrieffer, Theory of Superconductivity, Phys. Rev. 108, 1175 (1957).

[7] A. Macridin, P. Spentzouris, J. Amundson, and R. Harnik, Electron-Phonon Systems on a Universal Quantum Computer, Phys. Rev. Lett. 121, 110504 (2018).

[8] A. Cavalleri, Photo-Induced Superconductivity, Contemp. Phys. 59, 31 (2018).

[9] I. Garate, Phonon-Induced Topological Transitions and Crossovers in Dirac Materials, Phys. Rev. Lett. 110, 046402 (2013).

[10] P. Giannozzi et al., QUANTUM ESPRESSO: A Modular and Open-Source Software Project for Quantum Simulations of Materials, J. Phys. Condens. Matter 21, 395502 (2009).

[11] See Supplemental Material at http://link.aps.org/ supplemental/10.1103/PhysRevX.11.041060 for a discussion on the contributions of each parameter used in the numerical model.

[12] Si. Tanaka, M. Matsunami, and Si. Kimura, An Investigation of Electron-Phonon Coupling via Phonon Dispersion Measurements in Graphite Using Angle-Resolved Photoelectron Spectroscopy, Sci. Rep. 3, 3031 (2013).

[13] M.X. Na et al., Direct Determination of Mode-Projected Electron-Phonon Coupling in the Time Domain, Science 366, 1231 (2019). 
[14] P. Hein, S. Jauernik, H. Erk, L. Yang, Y. Qi, Y. Sun, C. Felser, and M. Bauer, Mode-Resolved Reciprocal Space Mapping of Electron-Phonon Interaction in the Weyl Semimetal Candidate, Nat. Commun. 11, 2613 (2020).

[15] H. Yan, D. Song, K. F. Mak, I. Chatzakis, J. Maultzsch, and T. F. Heinz, Time-Resolved Raman Spectroscopy of Optical Phonons in Graphite: Phonon Anharmonic Coupling and Anomalous Stiffening, Phys. Rev. B 80, 121403 (2009).

[16] J.-A. Yang, S. Parham, D. Dessau, and D. Reznik, Novel Electron-Phonon Relaxation Pathway in Graphite Revealed by Time-Resolved Raman Scattering and Angle-Resolved Photoemission Spectroscopy, Sci. Rep. 7, 40876 (2017).

[17] T. P. Devereaux et al., Directly Characterizing the Relative Strength and Momentum Dependence of Electron-Phonon Coupling Using Resonant Inelastic X-Ray Scattering, Phys. Rev. X 6, 041019 (2016).

[18] S. Tanaka, K. Mukai, and J. Yoshinobu, Direct Observation of the Electron-Phonon Coupling between Empty States in Graphite via High-Resolution Electron Energy-Loss Spectroscopy, Phys. Rev. B 95, 165408 (2017).

[19] T. R. Kirkpatrick and D. Belitz, Relevance of Many-Body Interactions for Correlated Electrons in the StrongCoupling Limit, Phys. Rev. B 69, 245122 (2004).

[20] A. Kar, L. Chen, and I. Franco, Understanding the Fundamental Connection between Electronic Correlation and Decoherence, J. Phys. Chem. Lett. 7, 1616 (2016).

[21] F. Giustino, M. L. Cohen, and S. G. Louie, Electron-Phonon Interaction Using Wannier Functions, Phys. Rev. B 76, 165108 (2007).

[22] M. Bernardi, First-Principles Dynamics of Electrons and Phonons*, Eur. Phys. J. B 89, 239 (2016).

[23] A. V. Belinskii and M. V. Chekhova, Photon Correlation in Inelastic Scattering of Light, Zh. Éksp. Teor. Fiz. 108, 1956 (1995) [J. Exp. Theor. Phys. 81, 1067 (1995)].

[24] O. V. Misochko, M. Tani, K. Sakai, K. Kisoda, S. Nakashima, V. N. Andreev, and F. A. Chudnovsky, Phonons in Above and Below the Mott Transition: A Comparison of Timeand Frequency-Domain Spectroscopy Results, Physica (Amsterdam) 263-264B, 57 (1999).

[25] F. Silva, S. M. Teichmann, S. L. Cousin, M. Hemmer, and J. Biegert, Spatiotemporal Isolation of Attosecond Soft X-Ray Pulses in the Water Window, Nat. Commun. 6, 6611 (2015).

[26] S. M. Teichmann, F. Silva, S. L. Cousin, M. Hemmer, and J. Biegert, 0.5-keV Soft X-Ray Attosecond Continua, Nat. Commun. 7, 11493 (2016).

[27] F. de Groot, Multiplet Effects in X-Ray Spectroscopy, Coord. Chem. Rev. 249, 31 (2005).

[28] M. Breusing, C. Ropers, and T. Elsaesser, Ultrafast Carrier Dynamics in Graphite, Phys. Rev. Lett. 102, 086809 (2009).

[29] G. Rohde, A. Stange, A. Müller, M. Behrendt, L.-P. Oloff, K. Hanff, T. J. Albert, P. Hein, K. Rossnagel, and M. Bauer, Ultrafast Formation of a Fermi-Dirac Distributed Electron Gas, Phys. Rev. Lett. 121, 256401 (2018).

[30] S. Piscanec, M. Lazzeri, F. Mauri, A. C. Ferrari, and J. Robertson, Kohn Anomalies and Electron-Phonon Interactions in Graphite, Phys. Rev. Lett. 93, 185503 (2004).

[31] M. Mohr, J. Maultzsch, E. Dobardžić, S. Reich, I. Milošević, M. Damnjanović, A. Bosak, M. Krisch, and C. Thomsen, Phonon Dispersion of Graphite by Inelastic X-Ray Scattering, Phys. Rev. B 76, 035439 (2007).
[32] T. Kampfrath, L. Perfetti, F. Schapper, C. Frischkorn, and M. Wolf, Strongly Coupled Optical Phonons in the Ultrafast Dynamics of the Electronic Energy and Current Relaxation in Graphite, Phys. Rev. Lett. 95, 187403 (2005).

[33] R. P. Chatelain, V. R. Morrison, B. L. M. Klarenaar, and B. J. Siwick, Coherent and Incoherent Electron-Phonon Coupling in Graphite Observed with Radio-Frequency Compressed Ultrafast Electron Diffraction, Phys. Rev. Lett. 113, 235502 (2014).

[34] M. Bernardi, D. Vigil-Fowler, J. Lischner, J. B. Neaton, and S. G. Louie, Ab Initio Study of Hot Carriers in the First Picosecond after Sunlight Absorption in Silicon, Phys. Rev. Lett. 112, 257402 (2014).

[35] M. Mihaila, D. Ursutiu, and I. Sandu, Electron-Phonon Coupling as the Source of 1/f Noise in Carbon Soot, Sci. Rep. 9, 947 (2019).

[36] J. H. Back, C.-L. Tsai, S. Kim, S. Mohammadi, and M. Shim, Manifestation of Kohn Anomaly in Fluctuations in Metallic Carbon Nanotubes, Phys. Rev. Lett. 103, 215501 (2009).

[37] E. G. Idrisov, Finite Frequency Noise in a Chiral Luttinger Liquid Coupled to Phonons, Phys. Rev. B 100, 155422 (2019).

[38] F. Carbone, The Interplay between Structure and Orbitals in the Chemical Bonding of Graphite, Chem. Phys. Lett. 496, 291 (2010).

[39] S. L. Cousin, N. Di Palo, B. Buades, S. M. Teichmann, M. Reduzzi, M. Devetta, A. Kheifets, G. Sansone, and J. Biegert, Attosecond Streaking in the Water Window: A New Regime of Attosecond Pulse Characterization, Phys. Rev. X 7, 041030 (2017).

[40] B. Buades et al., Dispersive Soft X-Ray Absorption FineStructure Spectroscopy in Graphite with an Attosecond Pulse, Optica 5, 502 (2018).

[41] Z. Xi, W. Su-Ju, Y. Wei-Wei, W. Hui, and L. C. D, Metrology of Time-Domain Soft X-Ray Attosecond Pulses and Reevaluation of Pulse Durations of Three Recent Experiments, Phys. Rev. Applied 13, 034043 (2020).

[42] M. Zürch et al., Direct and Simultaneous Observation of Ultrafast Electron and Hole Dynamics in Germanium, Nat. Commun. 8, 15734 (2017).

[43] F. Sette, G. K. Wertheim, Y. Ma, G. Meigs, S. Modesti, and C. T. Chen, Lifetime and Screening of the C Photoemission in Graphite, Phys. Rev. B 41, 9766 (1990).

[44] D. F. Escande, F. Doveil, and Y. Elskens, -Body Description of Debye Shielding and Landau Damping, Plasma Phys. Controlled Fusion 58, 014040 (2016).

[45] M. Schultze et al., Attosecond Band-Gap Dynamics in Silicon, Science 346, 1348 (2014).

[46] M. Lucchini, S. A. Sato, A. Ludwig, J. Herrmann, M. Volkov, L. Kasmi, Y. Shinohara, K. Yabana, L. Gallmann, and U. Keller, Attosecond Dynamical Franz-Keldysh Effect in Polycrystalline Diamond, Science 353, 916 (2016).

[47] H. Mashiko, K. Oguri, T. Yamaguchi, A. Suda, and H. Gotoh, Petahertz Optical Drive with Wide-Bandgap Semiconductor, Nat. Phys. 12, 741 (2016).

[48] F. Schlaepfer, M. Lucchini, S. A. Sato et al., Attosecond Optical-Field-Enhanced Carrier Injection into the GaAs Conduction Band, Nat. Phys. 14, 560 (2018). 
[49] M. Baudisch et al., Ultrafast Nonlinear Optical Response of Dirac Fermions in Graphene, Nat. Commun. 9, 1018 (2018).

[50] I. Gierz et al., Tracking Primary Thermalization Events in Graphene with Photoemission at Extreme Time Scales, Phys. Rev. Lett. 115, 086803 (2015).

[51] E. Malic, T. Winzer, F. Wendler, and A. Knorr, Review on Carrier Multiplication in Graphene, Phys. Status Solidi B 253, 2303 (2016).

[52] K. J. Tielrooij, J. C. W. Song, S. A. Jensen, A. Centeno, A. Pesquera, A.Z. Elorza, M. Bonn, L.S. Levitov, and F. H. L. Koppens, Photoexcitation Cascade and Multiple Hot-Carrier Generation in Graphene, Nat. Phys. 9, 248 (2013).

[53] I. Gierz, J. C. Petersen, M. Mitrano, C. Cacho, I. C. E. Turcu, E. Springate, A. Stöhr, A. Köhler, U. Starke, and A. Cavalleri, Snapshots of Non-Equilibrium Dirac Carrier Distributions in Graphene, Nat. Mater. 12, 1119 (2013).

[54] T. Winzer and E. Malić, Impact of Auger Processes on Carrier Dynamics in Graphene, Phys. Rev. B 85, 241404 (2012).

[55] L. Waldecker, T. Vasileiadis, R. Bertoni, R. Ernstorfer, T. Zier, F.H. Valencia, M.E. Garcia, and E.S. Zijlstra, Coherent and Incoherent Structural Dynamics in LaserExcited Antimony, Phys. Rev. B 95, 054302 (2017).

[56] K. Ishioka, M. Hase, M. Kitajima, L. Wirtz, A. Rubio, and H. Petek, Ultrafast Electron-Phonon Decoupling in Graphite, Phys. Rev. B 77, 121402 (2008).

[57] J. Weisshaupt, A. Rouzée, M. Woerner, M. J. J. Vrakking, T. Elsaesser, E. L. Shirley, and A. Borgschulte, Ultrafast Modulation of Electronic Structure by Coherent Phonon Excitations, Phys. Rev. B 95, 081101 (2017).

[58] B. Bauerhenne, V. P. Lipp, T. Zier, E. S. Zijlstra, and M. E. Garcia, Self-Learning Method for Construction of Analytical Interatomic Potentials to Describe Laser-Excited Materials, Phys. Rev. Lett. 124, 085501 (2020).

[59] S. Krylow, F. V. Hernandez, B. Bauerhenne, and M. E. Garcia, Ultrafast Structural Relaxation Dynamics of LaserExcited Graphene: Ab Initio Molecular Dynamics Simulations Including Electron-Phonon Interactions, Phys. Rev. B 101, 205428 (2020).

[60] L. M. Malard, M. A. Pimenta, G. Dresselhaus, and M. S. Dresselhaus, Raman Spectroscopy in Graphene, Phys. Rep. 473, 51 (2009).

[61] M. J. Stern, L. P. René de Cotret, M. R. Otto, R. P. Chatelain, J.-P. Boisvert, M. Sutton, and B. J. Siwick, Mapping Momentum-Dependent Electron-Phonon Coupling and Nonequilibrium Phonon Dynamics with Ultrafast Electron Diffuse Scattering, Phys. Rev. B 97, 165416 (2018).

[62] E. S. Zijlstra, L. L. Tatarinova, and M. E. Garcia, LaserInduced Phonon-Phonon Interactions in Bismuth, Phys. Rev. B 74, 220301 (2006).

[63] S. Krylow, E. S. Zijlstra, F. C. Kabeer, T. Zier, B. Bauerhenne, and M. E. Garcia, Nonequilibrium Dynamics of the Phonon Gas in Ultrafast-Excited Antimony, Phys. Rev. Mater. 1, 073601 (2017).

[64] D. Novko, F. Caruso, C. Draxl, and E. Cappelluti, Ultrafast Hot Phonon Dynamics in $\mathrm{MgB}_{2}$ Driven by Anisotropic Electron-Phonon Coupling, Phys. Rev. Lett. 124, 077001 (2020).
[65] S. L. Cousin, F. Silva, S. Teichmann, M. Hemmer, B. Buades, and J. Biegert, High-Flux Table-Top Soft X-Ray Source Driven by Sub-2-Cycle, CEP Stable, 1-kHz Pulses for Carbon K-Edge Spectroscopy, Opt. Lett. 39, 5383 (2014).

[66] F. Silva, S. M. Teichmann, S. L. Cousin, M. Hemmer, and J. Biegert, Spatiotemporal Isolation of Attosecond Soft X-Ray Pulses in the Water Window, Nat. Commun. 6, 6611 (2015).

[67] S. M. Teichmann, F. Silva, S. L. Cousin, M. Hemmer, and J. Biegert, 0.5-keV Soft X-Ray Attosecond Continua, Nat. Commun. 7, 11493 (2016).

[68] S. L. Cousin, Attosecond Streaking in the Water Window: A New Regime of Attosecond Pulse Characterization, Phys. Rev. X 7, 041030 (2017).

[69] E. Malic, T. Winzer, F. Wendler, and A. Knorr, Review on Carrier Multiplication in Graphene, Phys. Status Solidi B 253, 2303 (2016).

[70] W. R. L. Lambrecht et al., X-Ray Absorption, Glancing-Angle Reflectivity, and Theoretical Study of the $N \mathrm{~K}$-and $\mathrm{Ga} \mathrm{M}_{2,3}$ Edge Spectra in GaN, Phys. Rev. B 55, 2612 (1997).

[71] F. Dorchies et al., X-Ray Absorption K Edge as a Diagnostic of the Electronic Temperature in Warm Dense Aluminum, Phys. Rev. B 92, 085117 (2015).

[72] G. R. Stewart, Measurement of Low-Temperature Specific Heat, Rev. Sci. Instrum. 54, 1 (1983).

[73] E. Pop, V. Varshney, and A. K. Roy, Thermal Properties of Graphene: Fundamentals and Applications, MRS Bull. 37, 1273 (2012).

[74] D. S. Ivanov and L. V. Zhigilei, Combined AtomisticContinuum Modeling of Short-Pulse Laser Melting and Disintegration of Metal Films, Phys. Rev. B 68, 064114 (2003).

[75] B. Bauerhenne, V. P. Lipp, T. Zier, E. S. Zijlstra, and M. E. Garcia, Self-Learning Method for Construction of Analytical Interatomic Potentials to Describe Laser-Excited Materials, Phys. Rev. Lett. 124, 085501 (2020).

[76] E. S. Zijlstra, T. Zier, B. Bauerhenne, S. Krylow, P. M. Geiger, and M.E. Garcia, Femtosecond-Laser-Induced Bond Breaking and Structural Modifications in Silicon, $\mathrm{TiO}_{2}$ and Defective Graphene: An Ab Initio Molecular Dynamics Study, Appl. Phys. A 114, 1 (2014).

[77] J. P. Perdew and Y. Wang, Accurate and Simple Analytic Representation of the Electron-Gas Correlation Energy, Phys. Rev. B 45, 13244 (1992).

[78] E. S. Zijlstra, N. Huntemann, A. Kalitsov, M. E. Garcia, and U. von Barth, Optimized Gaussian Basis Sets for Goedecker-Teter-Hutter Pseudopotentials, Model. Simul. Mater. Sci. Eng. 17, 015009 (2009).

[79] S. Goedecker, M. Teter, and J. Hutter, Separable DualSpace Gaussian Pseudopotentials, Phys. Rev. B 54, 1703 (1996).

[80] C. Hartwigsen, S. Goedecker, and J. Hutter, Relativistic Separable Dual-Space Gaussian Pseudopotentials from $\mathrm{H}$ to Rn, Phys. Rev. B 58, 3641 (1998).

[81] M. Breusing, C. Ropers, and T. Elsaesser, Ultrafast Carrier Dynamics in Graphite, Phys. Rev. Lett. 102 (2009).

[82] M. Breusing, S. Kuehn, T. Winzer, E. Malić, F. Milde, N. Severin, J. P. Rabe, C. Ropers, A. Knorr, and T. Elsaesser, Ultrafast Nonequilibrium Carrier Dynamics in a Single Graphene Layer, Phys. Rev. B 83, 153410 (2011). 
[83] J. C. Johannsen et al., Direct View of Hot Carrier Dynamics in Graphene, Phys. Rev. Lett. 111, 027403 (2013).

[84] C. H. Lui, K. F. Mak, J. Shan, and T. F. Heinz, Ultrafast Photoluminescence from Graphene, Phys. Rev. Lett. 105, 127404 (2010).

[85] H. Wang et al., Ultrafast Relaxation Dynamics of Hot Optical Phonons in Graphene, Appl. Phys. Lett. 96, 081917 (2010).

[86] P. B. Allen, Theory of Thermal Relaxation of Electrons in Metals, Phys. Rev. Lett. 59, 1460 (1987).

[87] E. Runge and E. K. U. Gross, Density-Functional Theory for Time-Dependent Systems, Phys. Rev. Lett. 52, 997 (1984).

[88] J. K. Dewhurst, K. Krieger, S. Sharma, and E. K. U. Gross, An Efficient Algorithm for Time Propagation as Applied to
Linearized Augmented Plane Wave Method, Comput. Phys. Commun. 209, 92 (2016).

[89] J. K. Dewhurst, K. Krieger, P. Elliott, S. Sharma, and E. K. U. Gross, The ELK Code, http://elk.sourceforge.net/.

[90] K. Krieger, J. K. Dewhurst, P. Elliott, S. Sharma, and E. K. U. Gross, Laser-Induced Demagnetization at Ultrashort Time Scales: Predictions of TDDFT, J. Chem. Theory Comput. 11, 4870 (2015).

Correction: The given name of the 16th author contained an error and has been fixed. 